\title{
Preregistration Is Hard, And Worthwhile
}

\author{
Brian A. Nosek Center for Open Science; University of Virginia \\ Emorie D. Beck Washington University in St. Louis \\ Lorne Campbell University of Western Ontario \\ Jessica K. Flake McGill University \\ Tom E. Hardwicke Meta-Research Innovation Center Berlin (METRIC-B), QUEST Center for \\ Transforming Biomedical Research, Berlin Institute of Health, Charité - Universitätsmedizin \\ Berlin \\ David T. Mellor Center for Open Science \\ Anna E. van 't Veer Methodology and Statistics unit, Institute of Psychology, Leiden University \\ Simine Vazire University of California, Davis
}

Corresponding Author: Brian A. Nosek, nosek@virginia.edu

Keywords: Preregistration, exploratory research, confirmatory research, transparency, reproducibility

Conflict of Interest Disclosure: B. A. Nosek and D. T. Mellor are employees of the Center for Open Science a non-profit technology and culture change organization with a mission to increase openness, integrity, and reproducibility of research. All other authors declare no conflicts of interest.

Acknowledgements: This work was supported by grants from the National Institute of Aging, John Templeton Foundation, Templeton World Charity Foundation, Templeton Religion Trust, and Arnold Ventures to B. A. Nosek (PI). The Meta-Research Innovation Center Berlin (METRIC-B) is supported by a grant from the Einstein Foundation and Stiftung Charitè.

Contribution Statement: B. A. Nosek developed the initial outline and converted the outline into a draft manuscript. All authors contributed to the outline and editing and improving the draft. All authors reviewed the figure and text for final revisions. 


\section{Abstract (49/50)}

Preregistration clarifies the distinction between planned and unplanned research by reducing unnoticed flexibility. This improves credibility of findings and calibration of uncertainty. However, making decisions before conducting analyses requires practice. During report writing, respecting both what was planned and what actually happened requires good judgment and humility in making claims.

Preregistration of studies serves at least three aims for improving the credibility and reproducibility of research findings (Nosek et al., 2018; van 't Veer \& Giner-Sorolla, 2016; Wagenmakers et al., 2012). First, preregistration of analysis plans makes clear which analyses were planned a priori and which were conducted post hoc. This improves calibration of uncertainty for unplanned analyses, and diagnosticity of statistical inferences for planned analyses. Most often, planned analyses are equated with hypothesis testing or confirmatory research, but one can also preregister analysis plans when there are no a priori hypotheses to test--i.e., a planned exploratory analysis. Doing so has the benefit of strengthening statistical inferences as compared with unplanned analyses. Statistical tests always involve uncertainty: e.g., at a criterion value of $5 \%$, the presumed Type I error rate is $5 \%$. However, this holds only if data meet the assumptions for a single statistical test. Undisclosed and unplanned analyses undermine the assumptions. Preregistration makes transparent the uncertainty of statistical tests by showing how many statistical tests were conducted and enabling an accurate familywise error rate.

Second, preregistration enables detection of questionable research practices such as selective outcome reporting (John et al. 2012) or Hypothesizing After the Results are Known (HARKing; Kerr, 1998). Third, preregistration of studies can reduce the impact of publication bias-particularly the prioritization of publishing positive over negative results-by making all planned studies discoverable whether or not they are ultimately published.

These benefits are evident with rapid growth in use of preregistration to improve research rigor (Nosek \& Lindsay, 2018). However, preregistration is a skill that requires experience to hone. Getting the most out of preregistration requires practice because the previous training of many scientists has involved making some important design and analysis decisions during analysis (John et al. 2012; Bem, 2004). Furthermore, whilst the dominant incentive structures of the scientific ecosystem promote intellectual defensiveness (Nosek, Spies, \& Motyl, 2012), preregistration may promote intellectual humility and encourage better calibration of scientific claims. With the accelerating adoption of preregistration, we now face the challenge of figuring out how to use this methodology to its fullest potential.

\section{Preregistration Improves with Practice}

It is common for researchers to begin a study with a general sense of how the methodology will be implemented, how the hypotheses will be tested, what exclusion rules will be applied, how variables will be combined, and which model form, covariates, and characteristics will be used. However, "a general sense" inevitably provides flexibility in making consequential decisions that could influence study execution, analysis, and reporting (Silberzahn et al., 2018; Simmons, Nelson, \& Simonsohn, 2011). Effective preregistration 
requires converting that general sense into precise, explicit plans that anticipate what has not yet occurred and decisions about what will be done. For example, when collecting new data, how will you decide when to stop data collection? During analysis, what are the specific steps needed to examine the questions of interest? During authoring, what outcomes will be reported? Making these decisions before the data are available is challenging, especially the first time. It can also provide the jarring experience that decisions made earlier in one's career had been more data contingent than recognized. In preregistration, the common comfort of having the decision process unfold as one completes the analysis is converted to an uncomfortable mental simulation of what decisions will need to be made eventually. Moreover, research rarely goes precisely according to plan. Data collection can take longer than anticipated; a skewed data distribution may require adjustments to the planned analysis; unanticipated outliers may not be addressed by prespecified exclusion criteria. When the outcomes are known, the universe of contingencies is small; when the outcomes are unknown, the universe of contingencies is much larger.

Preregistration requires research planning and it is hard, especially contingency planning. It takes practice to make design and analysis decisions in the abstract, and it takes experience to learn what contingencies are most important to anticipate. This might lead researchers to shy away from preregistration for worries about imperfection. Embrace incrementalism. Preregistration is a methodological skill that takes time to develop. Having some plans is better than having no plans, and sharing those plans in advance is better than not sharing them. With experience, planning will improve and the benefits will increase for oneself and for consumers of the research.

There are opportunities to accelerate that skill building. Study registries such as OSF (http://osf.io/prereg/) and SREE (https://sreereg.icpsr.umich.edu/), and decision tools such as Declare Design (http://declaredesign.org/) provide structured workflows to help researchers anticipate common decisions and provide guidance for articulating those decisions. The more comprehensive workflows provide guidance through each part of the study from stimuli selection and presentation, research design and manipulation, measure selection and use, data processing and management, primary and secondary analyses, and interpretation of results. Some evidence suggests that such workflows are effective in improving the specificity of preregistrations (Veldkamp et al., 2018).

Other strategies for developing these skills include: (1) refining analysis plans by simulating data to practice making the decisions; (2) splitting the "real" data into exploratory and confirmatory subsamples and preregistering the analysis after viewing the exploratory subset; (3) drafting the study methods and results section in advance to help anticipate what should be done and how you will report it; and (4) submitting the plan as a Registered Report for peer review to get expert feedback on the plan.

Researchers can embrace the common back-and-forth between planned and unplanned (confirmatory and exploratory) research activities and use post hoc analyses as an opportunity to learn, which facilitates better planning in the next investigation. Over time, the iterative experience between planning and resolving unplanned contingencies can be translated into documented, standard operating procedures that outline default design and analysis decisions (Lin \& Green, 2016). In sum, there is value in unplanned discoveries despite their uncertainty 
and there is value in planned tests because of their diagnositicity. The key role of preregistration is to clarify which is which.

\section{Transparently Reporting Deviations Helps Calibrate Confidence in Claims}

Preregistration is a plan, not a prison (DeHaven, 2017). When deviations from the plan will improve the quality of the research, deviate from the plan. Many studies will have some deviations between the preregistered plan and what actually occurs. Planned analyses may contain errors or better approaches may emerge because of learning, discovery, and innovation. Obviously, deviations introduce risk. A seemingly trivial deviation might be highly consequential. For example, changing from a combined outcome variable to using just one component of the combination can inflate false positive risk (Simmons et al., 2011). Deviations inevitably make it harder to interpret with confidence what occurred in relation to what was planned. Transparency is key-all deviations from the plan should be acknowledged.

Reporting deviations from the plan can be challenging, especially because of journal word limits, pressures for narrative coherence, and reviewer expectations. If possible, report what occurs following the original plan alongside what occurs with the deviations, and share the materials, data, and code so that others can evaluate the reported outcomes and what would have occurred with alternative approaches. Transparency enables clarifying decisions across the research process, and facilitates others' understanding of justifications for those decisions.

Additional transparency strategies to maximize credibility of reported findings include: (1) When possible, update the preregistration with deviations before observing the data (e.g., fix errors in the analysis plan; add obvious exclusion rules that become apparent); (2) Mention all planned analyses, if only to explain why a planned analysis was not reported; (3) Include a table documenting and explaining all deviations; (4) Use supplements to share in full, not to hide inconvenient information; (5) During analysis, actively log the decisions as they made with rationales.

\section{Intellectual humility improves research credibility}

A successful program of research involves constantly striving to minimize error and misunderstanding while simultaneously recognizing that error and misunderstanding are inevitable. Research is hard, and scientists are fallible. Progress is made by identifying and addressing the shortcomings of yesterday's theories and methods to offer an improved understanding for tomorrow's skeptics to critique.

Preregistration can reveal the inevitable shortcomings of our research and consequently fosters intellectual humility. By embracing the fact that our present understanding is surely imperfect, identification of error presents an opportunity, not a threat. Preregistration provides opportunity for you and others to understand how you arrived at your claims. This means that you and others will be better positioned to find the weaknesses of what you planned and also identify ways to do it better the next time. Making the most of preregistration will require that we reduce the ego-driven desire to "be right" and cultivate the more productive truth-seeking desire to "get it right" (Ebersole et al., 2016).

[1448/1500 words] 
Box: Getting Started with Preregistration

Articles providing conceptual and practical information about preregistration:

- "A Manifesto for Reproducible Science" Munafo et al., 2017

- "The Preregistration Revolution" Nosek et al., 2018

- "An agenda for purely confirmatory research" Wagenmakers et al., 2012

- "Pre-registration in Social Psychology—a discussion and suggested template" van 't Veer \& Giner-Sorolla, 2016

Registration workflows with relatively comprehensive guidance for decision-making:

- Clinicaltrials.gov: http://clinicaltrials.gov/

- OSF: http://osf.io/prereg/

- SREE: https://sreereg.icpsr.umich.edul

Finding primers, help guides, and other resources:

- https://cos.io/prereg/ 


\section{References (15/15)}

Bem, D. J. (2004). Writing the empirical journal article. In J. M. Darley, M. P. Zanna, \& H. L. Roediger, III (Eds.), The compleat academic: A career guide (2nd ed., pp. 185-219). Washington, DC: American Psychological Association.

DeHaven, A. (2017). Preregistration: A Plan, Not a Prison. Retrieved from https://cos.io/blog/preregistration-plan-not-prison/

Ebersole, C. R., Axt, J. R., \& Nosek, B. A. (2016). Scientists' reputations are based on getting it right, not being right. PLOS Biology, 14, e1002460. Doi: 10.1371/journal.pbio.1002460

John, L. K., Loewenstein, G., \& Prelec, D. (2012). Measuring the prevalence of questionable research practices with incentives for truth telling. Psychological Science, 23, 524-532.

Kerr, N. L. (1998). HARKing: Hypothesizing After the Results are Known. Personality and Social Psychology Review, 2, 196-217.

Lin, W., \& Green, D. P. (2016). Standard operating procedures: A safety net for pre-analysis plans. PS: Political Science \& Politics, 49, 495-500. Doi: 10.1017/S1049096516000810

Munafò, M. R., Nosek, B. A., Bishop, D. V. M., Button, K. S., Chambers, C. D., Percie du Sert, N., Simonsohn, U., Wagenmakers, E-J., Ware, J. J., \& loannidis, J. P. A. (2017). A manifesto for reproducible science. Nature Human Behavior, 1, 0021. Doi: 10.1038/s41562-016-0021

Nosek, B. A., Ebersole, C. R., DeHaven, A., Mellor, D. M. (2018). The preregistration revolution. Proceedings for the National Academy of Sciences, 115, e10518. Doi:

10.1073/pnas. 1816418115

Nosek, B. A., \& Lindsay, D. S. (2018). Preregistration becoming the norm in psychological science. APS Observer, 31(30/3).

Nosek, B. A., Spies, J. R., \& Motyl, M. (2012). Scientific utopia: II. Restructuring incentives and practices to promote truth over publishability. Perspectives on Psychological Science, 7, 615631. DOI: $10.1177 / 1745691612459058$

Silberzahn, R., Uhlmann, E. L., Martin, D. P., Anselmi, P., Aust, F., Awtrey, E. C., ... \& Nosek, B. A. (2018). Many analysts, one dataset: Making transparent how variations in analytical choices affect results. Advances in Methods and Practices in Psychological Science, 1, 337-356. Doi: 10.1177/2515245917747646

Simmons, J. P., Nelson, L. D., \& Simonsohn, U. (2011). False-positive psychology undisclosed flexibility in data collection and analysis allows presenting anything as significant. Psychological Science, 22, 1359-1366. 
van 't Veer, A. E., \& Giner-Sorolla, R. (2016). Pre-registration in Social Psychology-a discussion and suggested template. Journal of Experimental Social Psychology, 67, 2-12. doi:10.1016/j.jesp.2016.03.004

Veldkamp, C. L. S., Bakker, M., van Assen, M. A. L. M., Crompvoets, E. A. V., Ong, H. H., Soderberg, C. K., Mellor, D., Nosek, B. A., \& Wicherts, J. M. (2018). Ensuring the quality and specificity of preregistrations. https://psyarxiv.com/cdgyh/ Doi: 10.31234/osf.io/cdgyh

Wagenmakers, E. J., Wetzels, R., Borsboom, D., van der Maas, H. L. J., \& Kievit, R. A. (2012). An agenda for purely confirmatory research. Perspectives on Psychological Science, 7, 632638. 